\title{
A SURVEY OF STACKELBERG DIFFERENTIAL GAME MODELS IN SUPPLY AND MARKETING CHANNELS
}

\author{
Xiuli HE $^{\mathbf{1}}$ Ashutosh PRASAD $^{\mathbf{1}}$ Suresh P. SETHI ${ }^{1}$ Genaro J. GUTIERREZ ${ }^{2}$ \\ ${ }^{1}$ School of Management, The University of Texas at Dallas, Richardson, TX 75080 \\ xiulihe@utdallas.edu,aprasad@utdallas.edu,sethi@utdallas.edu $(\square)$ \\ 2McCombs School of Business, The University of Texas at Austin, Austin, TX 78731 \\ genarojg@mail.utexas.edu
}

\section{Erratum to: J Syst Sci Syst Eng \\ DOI: 10.1007/s11518-007-5058-2}

The presentation of Table 2 in the original version of this article contained a few typos. The corrected Table 2 is given below.

Table 2 Summary of model descriptions

\begin{tabular}{lllll}
\hline Paper & Dynamics & L's decision & F's decisions & Solution ${ }^{1}$ \\
\hline Breton, Jarrar, and Zaccour (2006) & Lanchester type & Ad. effort & Ad. effort & FS \\
Desai (1992) & Seasonal & Price, Production rate & Price & OLS \\
Desai (1996) & Seasonal & Price, Production rate & Price & OLN,FN,OLS \\
Eliashberg and Steinberg (1987) & Seasonal & Price, Production rate & Price & OLS \\
Gutierrez and He (2007) & Bass type & Price & Price & OLS \\
He, Prasad, and Sethi (2007) & Sethi model & Price, Particip. rate & Price, Ad. effort & FS \\
He and Sethi (2008) & Bass type & Price & Price, Shelf spaceOLS \\
JSZ (2000) & NA model & Ad. Effort, Particip. rate & Ad. effort & FS \\
JSZ (2001) & NA model & Price, Ad. effort & Price, Ad. effort & FN, FS \\
JTZ (2003) & NA model & Ad. Effort, Particip. rate & Price, Ad. effort & FN,FS \\
JTZ (2006) & NA model & Ad. effort & Ad. effort & FS \\
Karray and Zaccour (2005) & NA model & Price, Ad. effort & Price, Ad. effort & FS \\
Kogan and Tapiero (2007a) & Linear & Price, Production rate & Price & OLS \\
Kogan and Tapiero (2007b) & Linear & Processing rate & Production rate & OLS \\
Kogan and Tapiero (2007c) & Linear & Price & Order quantity & OLS \\
Kogan and Tapiero (2007d) & Linear & Price & Order quantity & OLS \\
Kogan and Tapiero (2007e) & Linear & Price & Price & OLS \\
Kogan and Tapiero (2007f) & Linear & Price & Price & OLN,OLS \\
Martin-Herran and Taboubi (2005) NA model & Ad. effort, Incentive & Shelf space & FN, FS \\
\hline
\end{tabular}

${ }^{1}$ The symbol OLN = Open-loop Nash equilibrium, FN = Feedback Nash equilibrium, OLS= Open-loop Stackelberg equilibrium, and FS = Feedback Stackelberg equilibrium.

${ }^{2} \mathrm{JSZ}$ is the abbreviation for Jogensen, Sigue, and Zacccour.

${ }^{3} \mathrm{JTZ}$ is the abbreviation for Jogensen, Taboubi, and Zaccour. 